in vivo $34: 3163-3169(2020)$

doi:10.21873/invivo.12151

\title{
Response of Triple-negative Breast Cancer Liver Metastasis to Oral Recombinant Methioninase in a Patient-derived Orthotopic Xenograft (PDOX) Model
}

\author{
HYE IN LIM ${ }^{1,2,3}$, JUN YAMAMOTO ${ }^{1,2}$, QINHONG HAN ${ }^{1}$, YU SUN ${ }^{1,2}$, HIROTO NISHINO ${ }^{1,2}$, \\ YOSHIHIKO TASHIRO ${ }^{1,2}$, NORIHIKO SUGISAWA ${ }^{1,2}$, YUYING TAN ${ }^{1}$, \\ HEE JUN CHOI ${ }^{4}$, SEOK JIN NAM ${ }^{5}$, MICHAEL BOUVET ${ }^{2}$ and ROBERT M. HOFFMAN ${ }^{1,2}$ \\ ${ }^{1}$ AntiCancer Inc, San Diego, CA, U.S.A.; \\ ${ }^{2}$ Department of Surgery, University of California, San Diego, CA, U.S.A.; \\ ${ }^{3}$ Department of Surgery, Chinjujeil Hospital, Jinju, Republic of Korea; \\ ${ }^{4}$ Department of Surgery, Samsung Changwon Hospital, \\ Sungkyunkwan University School of Medicine, Changwon, Republic of Korea; \\ ${ }^{5}$ Division of Breast Surgery, Department of Surgery, Samsung Medical Center, \\ Sungkyunkwan University School of Medicine, Seoul, Republic of Korea
}

\begin{abstract}
Background/Aim: The aim of this study was to establish a patient-derived orthotopic xenograft (PDOX) mouse model of liver metastasis of triple-negative breast cancer (TNBC) and examine the efficacy of oral recombinant methioninase (o-rMETase) on the liver metastasis. Materials and Methods: TNBC from a patient was implanted in the left hepatic lobe of nude mice to simulate liver metastasis in a PDOX model. Ten days later, all mice underwent laparotomy to measure tumor size and were randomized to three groups: control; o-rMETase 100 $U$ once daily (qd); and o-rMETase 200 U qd. After 9 days of treatment, all mice were sacrificed. Results: At the end of the treatment period for the liver metastasis, the size of liver metastases was $372.6 \mathrm{~mm}^{3}$ in the control group; $160.0 \mathrm{~mm}^{3}$ in the o-rMETase $100 \mathrm{U}$ group; and $245.3 \mathrm{~mm}^{3}$ in the orMETase $200 \mathrm{U}$ group. All mice had ascites and 12 out of 14 mice in all groups had mesenteric lymph-node metastasis, as re-metastasis. The mean body-condition score was 1.5 in the control group; 2.4 in the o-rMETase $100 \mathrm{U}$
\end{abstract}

This article is freely accessible online.

Correspondence to: Robert M. Hoffman, Ph.D., AntiCancer Inc, 7917 Ostrow St, San Diego, CA, 92111, U.S.A. Tel: +1 8586542555, Fax: +1 8582684175, e-mail: all@anticancer.com; Hye In Lim, MD, Department of Surgery, Chinjujeil Hospital, 885 Jinju-daero, Jinju-si, Gyeongsangnam-do, Republic of Korea, e-mail: vastprogress@naver.com

Key Words: PDOX, patient-derived orthotopic xenograft, TNBC, triple-negative breast cancer, liver metastasis, re-metastasis, lymph node, ascites, oral recombinant methioninase, treatment. group; and 2.6 in the o-rMETase 200 U group (control group vs. o-rMETase 200 U group, $p<0.05)$. Conclusion: The TNBC liver metastasis was highly aggressive resulting in re-metastasis and ascites. o-rMETase tended to inhibit the liver metastasis and significantly improved the mouse bodycondition score. This new PDOX model of TNBC liver metastasis will be useful for identifying effective agents for this recalcitrant disease.

Triple-negative breast cancer (TNBC) accounts for about $15 \sim 20 \%$ of breast cancers. TNBC grows and spreads faster and has higher risk of early relapse with visceral metastasis compared to other subtypes of breast cancer. The 5-year relative survival rate of TNBC with distant metastasis is only $11 \%$ and there is rapid progression from distant recurrence to death (1). Because TNBC with distant metastasis has a higher frequency of progression, efforts to identify effective treatments for metastatic TNBC are necessary.

Cancer cells are methionine addicted due to enhanced overall rates of transmethylation and therefore depend on high levels of methionine compared to normal cells (2-5). The high methionine/methylation flux of cancer cells is known as the Hoffman effect (6), analogous to the Warburg effect of glucose overuse by cancer cells. Our laboratory discovered methionine-addiction of cancer (2-5). We have studied this phenomenon for almost 50 years and have concluded that methionine-addiction is the most fundamental and general hallmark of cancer (3-5). Methionine-addiction is tightly linked to global epigenetic changes in cancer controlled by methylation events (7) and is a promising target of cancer treatment. 


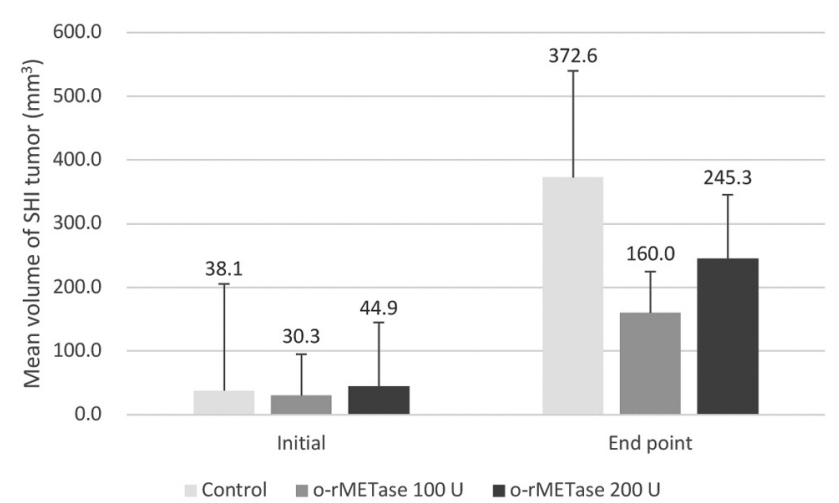

Figure 1. Efficacy of o-rMETase on TNBC liver metastasis. SHI: surgical hepatic implantation, o-rMETase: oral recombinant methioninase, TNBC: triple negative breast cancer. Error bars show standard error of the mean (SEM).

Methionine restriction selectively traps cancer cells in the $\mathrm{S} / \mathrm{G}_{2}$-phase of the cell cycle (8), where they are susceptible to most cytotoxic chemotherapy and can be successfully eradicated $(2,9)$. Recombinant methioninase, administered orally (o-rMETase), has shown efficacy in many solid tumors, for example, sarcoma (10-11), pancreatic cancer (12), colon cancer $(13,14)$, and malignant melanoma (15, 16) in patient-derived orthotopic xenograft (PDOX) mouse models, by restricting methionine.

We previously established liver-metastasis models of patient-derived colon cancer in nude mice (17-19). Lymphnode metastasis was found at the site of drainage of the liver: celiac, portal and mediastinal lymph nodes which originated from the liver metastasis, and not, as previously thought, from primary colon cancer. We suggested the concept of re-metastasis which means "metastasis of metastases" (18).

We previously established a patient-derived orthotopic xenograft (PDOX) model of highly-aggressive TNBC transplanted to the mammary fat pad of nude mice (20). In the present study, we established an aggressive TNBC livermetastasis model by surgical hepatic implantation (SHI) in nude mice and evaluated the efficacy of o-rMETase.

\section{Materials and Methods}

Mice. Athymic nu/nu nude, 4-6 weeks old female mice (AntiCancer Inc., San Diego, CA, USA), were used in this study. All animal studies were carried out with an AntiCancer Institutional Animal Care and Use Committee (IACUC)-protocol approved for this study and according to the procedures and principles in the National Institutes of Health Guide for the Care and Use of Animals under Assurance Number A3873-1. Housing, diet, anesthesia of animals have been described in detail in a previous study (20).

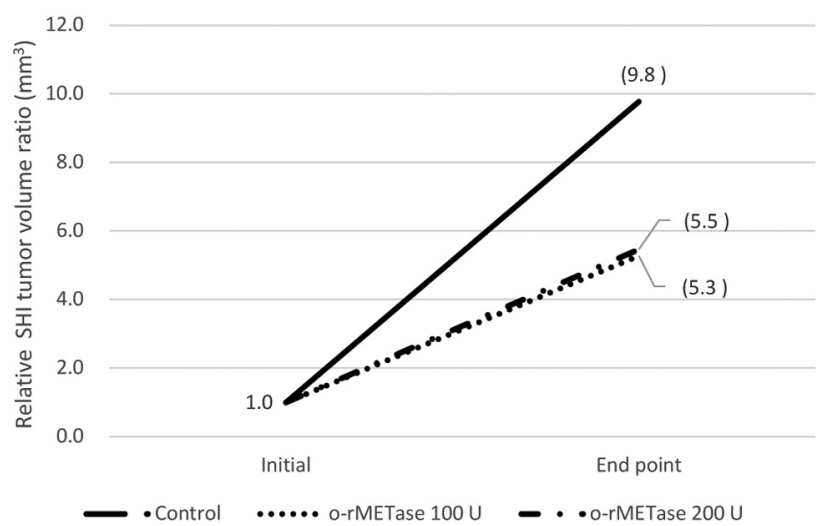

Figure 2. Comparison of relative tumor volume of SHI tumor with and without o-rMETase treatment. SHI: surgical hepatic implantation, orMETase: oral recombinant methioninase. Relative tumor volume (values in parentheses) is the ratio of the tumor volume at the endpoint compared to the initial tumor volume.

o-rMETase production and formulation. The L-methionine- $\gamma$ deamino- $\alpha$-mercaptomethane-lyase gene from Pseudomonas putida has been cloned in E. coli. o-rMETase is produced in three steps from the recombinant $E$. coli, including fermentation, purification and formulation. The fermentation procedure of the host $E$. coli cells, the purification protocol and formulation of rMETase have been previously described (21).

Patient-derived TNBC and establishment of PDOX. A 74-year-old female patient was diagnosed with TNBC in the right breast. She underwent breast-conserving surgery with sentinel lymph-node biopsy in at the Department of Surgery, Samsung Medical Center (SMC), Seoul, Korea. The tumor $(2.4 \mathrm{~cm})$ was an invasive ductal carcinoma with histological grade 3 and an $80 \%$ Ki-67 value. Regional and distant metastasis were not detected.

Written informed consent was obtained from the patient, and the Institutional Review Board (IRB) of SMC approved this experiment. We established the TNBC in nude mice as previously described (20). In the present study, when subcutaneously-grown tumors reached $10 \mathrm{~mm}$ in diameter, they were harvested and cut into approximately $1 \mathrm{~mm}^{3}$ size fragments. For surgical hepatic implantation (SHI), the abdomen was sterilized with $70 \%$ alcohol and a left para-median incision was made under anesthesia. After the left lobe of the liver was exteriorized, a shallow $2 \mathrm{~mm}$-length incision was made with an Iris surgical scissors on the serosa of the left-lateral lobe. The tumor fragment was inserted into this incision and followed by bleeding control with compression. The abdominal wall was closed with a 6-0 nylon suture.

Treatment dose and schedule. Ten days after SHI, mice underwent laparotomy to observe the tumor size on the liver. The mice were randomized into three groups of equivalent average tumor size: G1, untreated control [ $\mathrm{n}=4$, PBS $0.1 \mathrm{ml}$, per os (p.o.), twice a day, 9 consecutive days]; G2, $100 \mathrm{U}$ o-rMETase treatment ( $\mathrm{n}=5,50$ units, p.o., twice a day, 9 consecutive days); and G3, 200 U o-rMETase treatment ( $\mathrm{n}=5,100$ units, p.o., twice a day, 9 consecutive days). Treatment was started two days after laparotomy. All mice were humanely sacrificed on the following day of the last treatment. 


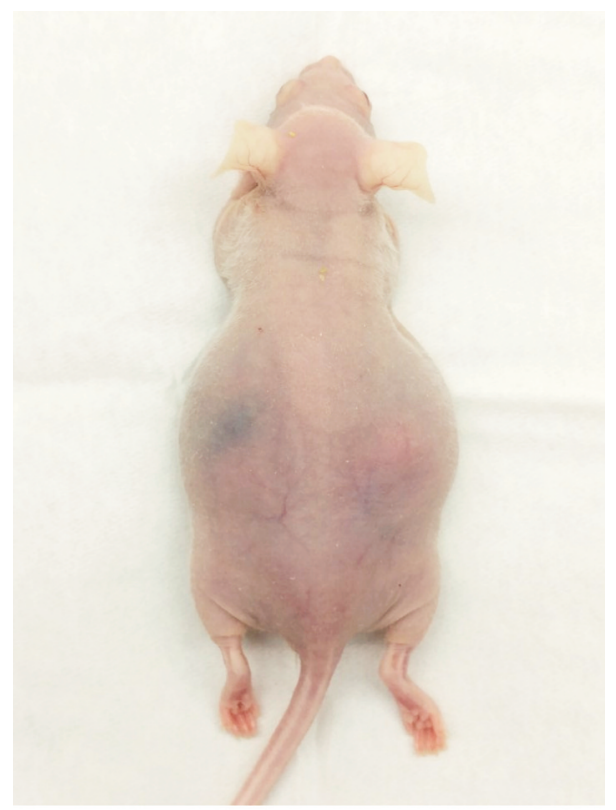

Figure 3. Mouse with ascites in control group.

Mouse body weight was measured every day or two and tumor volume was measured on the day of laparotomy and on the day of sacrifice. Tumor volume was calculated using the following formula: Tumor volume $\left(\mathrm{mm}^{3}\right)=$ length $(\mathrm{mm}) \times$ width $(\mathrm{mm}) \times$ width $(\mathrm{mm})$ $\times 1 / 2(22)$. Mouse condition was determined using body-condition scoring (BCS) (23), and behavior and appearance scoring (24).

\section{Results}

We implanted tumors to the liver of 22 mice using SHI. On the day of laparotomy before treatment, 14 mice had liver tumors and the mean tumor volume was $38.1 \mathrm{~mm}^{3}$ in the untreated control group; $30.3 \mathrm{~mm}^{3}$ in the o-rMETase $100 \mathrm{U}$ treatment group; and $44.9 \mathrm{~mm}^{3}$ in the o-rMETase $200 \mathrm{U}$ treatment group. After 9 days of treatment, the mean tumor volume of the liver was $372.6 \mathrm{~mm}^{3}$ in the control group; $160.0 \mathrm{~mm}^{3}$ in the o-rMETase $100 \mathrm{U}$ treatment group; and $245.3 \mathrm{~mm}^{3}$ in the o-rMETase $200 \mathrm{U}$ treatment group (Figure $1)$. The tumor volume ratio at the end of the treatment period relative to beginning was 9.8 in the control group; 5.3 in the o-rMETase $100 \mathrm{U}$ group; and 5.5 in the o-rMETase $200 \mathrm{U}$ group (Figure 2).

All mice had ascites (Figure 3) and 12 out of 14 mice had mesenteric metastatic lymph-node metastasis (Figure 4), except one each in the control and o-rMETase $100 \mathrm{U}$ group.

The mean behavioral and appearance scores were 5.5 in the control group; 9.6 in the o-rMETase $100 \mathrm{U}$ group; and 10 in the o-rMETase $200 \mathrm{U}$ group (Figure 5A). The mean BCS was 1.5 in the control group; 2.4 in the o-rMETase 100

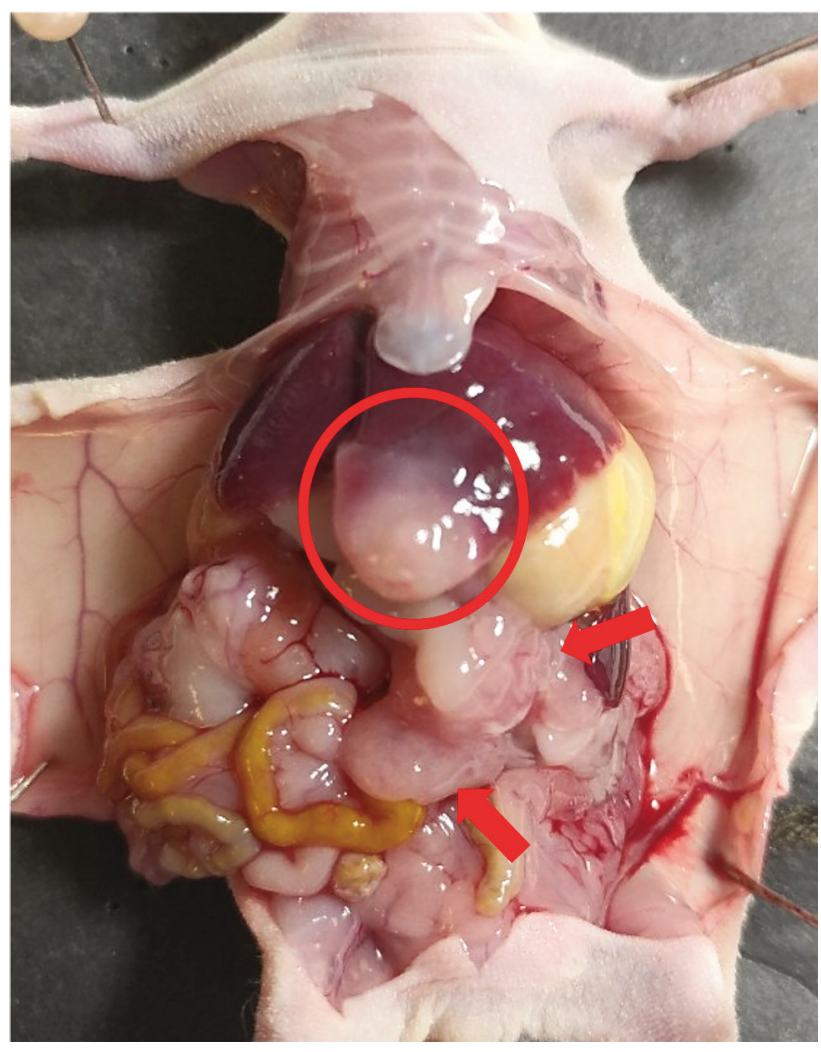

Figure 4. Tumor and lymph-node re-metastasis in TNBC SHI model on the day following the last treatment. Red circle is SHI tumor and red arrows are lymph-node re-metastasis. TNBC: Triple-negative breast cancer, SHI: surgical hepatic implantation.

U group; and 2.6 in the o-rMETase $200 \mathrm{U}$ group at the end point (control group vs. o-rMETase $200 \mathrm{U}$ treatment group, $p<0.05$ ) (Figure 5B).

There was no significant difference in body weight between groups, but o-rMETase $200 \mathrm{U}$ may have prevented cachexia. (Figure 6).

Hematoxylin and eosin staining showed cancer cells in both the lymph-node and liver metastasis (Figure 7).

\section{Discussion}

Mouse models of breast-cancer metastasis to the liver use human breast-cancer cell lines. Since liver metastasis rarely occurs in subcutaneous or orthotopic xenograft models of breast cancer, intracardiac or intrasplenic injection is used, but these models are not specific and can cause concurrent metastases in other organs (25). We previously established liver-metastasis models of patient-derived colon cancer in nude mice using SHI (17-19) and discovered the phenomenon of re-metastasis (18). In the present study, we established a liver-metastasis model of patient-derived TNBC by SHI, 


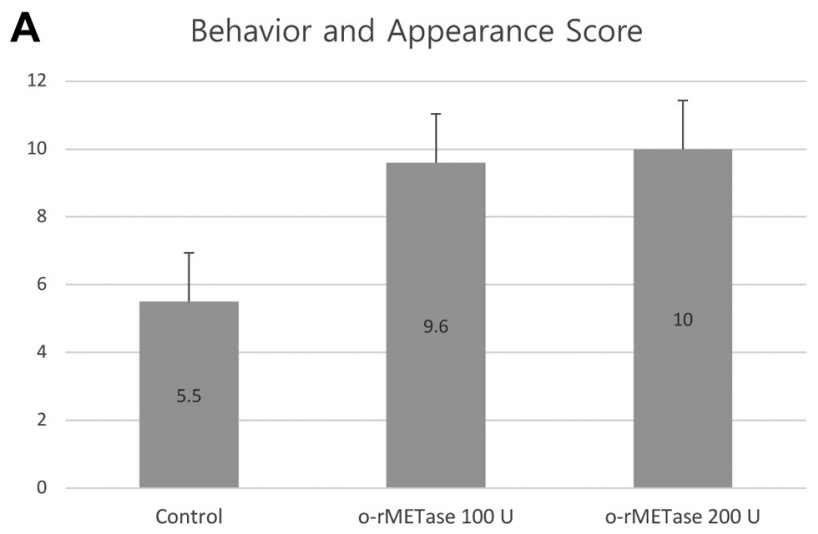

B

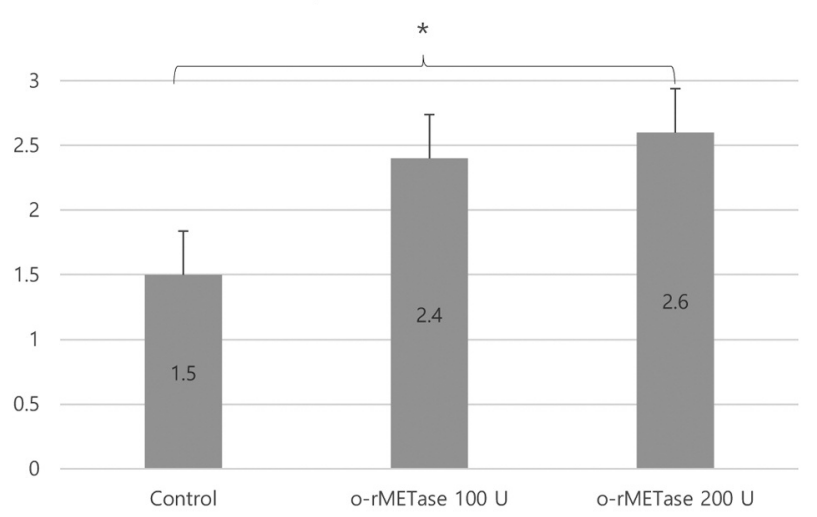

Figure 5. Comparison of body scoring of control and o-rMETase-treated mice. (A) Behavioral and appearance score at the end point. This score is quantified appearance, natural behavior, provoked behavior and ranged from 1 to 13 (23). (B) BCS at the end point. BCS is quantified by the amount of flesh covering body protuberances and raged from 1 to 5 (24). Error bars show standard error of the mean (SEM). o-rMETase: Oral recombinant methioninase, BCS: body condition score, * $p<0.05$.

simulating liver metastasis. In this SHI model, we found mesenteric lymph-node metastasis following liver metastasis growth, as a result of re-metastasis. Re-metastasis should be considered clinically when we treat metastatic disease.

TNBC has an increased likelihood of distant recurrence compared to other types of breast cancer and the risk of distant recurrence peaks between 1 to 3 years after surgery. The time from recurrence to death is 9 months, significantly shorter than other types of breast cancer (1). Because TNBC has no targetable marker, such as hormonal receptors or human epidermal growth factor receptor-2 (HER-2), the treatment options for metastatic disease are limited and novel treatment is necessary.

Compared to normal cells, cancer cells require high levels of methionine to proliferate due to methioninase addiction (25). Methionine addiction is cancer-specific metabolism and

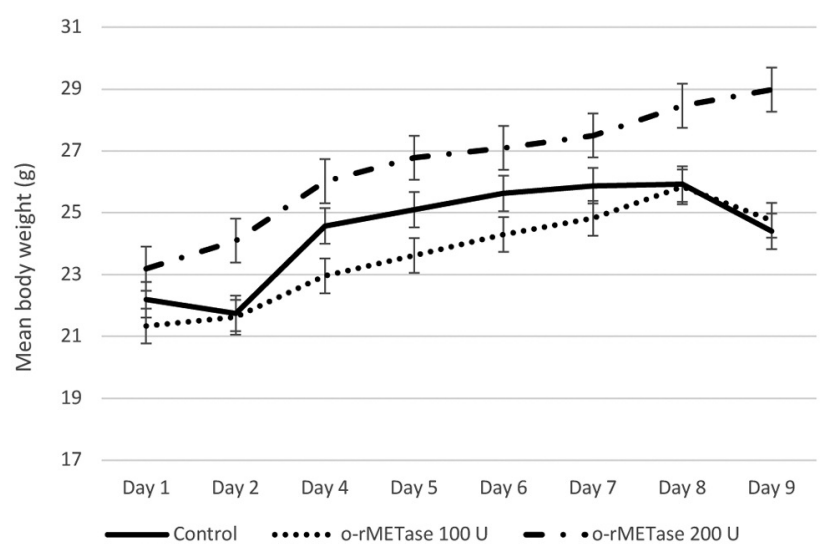

Figure 6. Comparison of body weight over time during treatment. Error bars show the standard error of the mean (SEM).

distinguishes cancer cells from normal cells. Targeting cancerspecific altered methionine metabolism has potential as a clinical cancer treatment (9) and can be a new effective treatment modality of TNBC. Methionine-restriction has been studied in a TNBC mouse model by Jeon et al. (26) who reported that a low-methionine diet inhibited TNBC metastasis in mice. Strekalova et al. (27) reported that dietary methionine deprivation enhanced the anti-tumor efficacy of a humanized agonistic TNF-related apoptosis-inducing ligand receptor-2 (TRAIL-R2) monoclonal antibody by increasing TRAIL-R2 expression in a model of TNBC.

In the present study, o-rMETase trended to inhibit the growth of the SHI tumors and resulted in better mouse condition. These results suggest that o-rMETase has potential as a new effective modality for metastatic TNBC, especially since it can be administered orally without toxicity $(28,29)$.

Our future studies will involve administration o-rMETase in combination with conventional and experimental chemotherapy for TNBC metastases using PDOX mouse models $(10-16,29)$. o-rMETase has already shown promise in the clinic (28) and will soon be tested on TNBC patients, especially with liver metastasis. o-rMETase has previously shown efficacy to inhibit post-surgical recurrence of TNBC in a PDOX nude-mouse model (30). This new PDOX model of TNBC liver metastasis has potential to identify curative therapeutics for this recalcitrant disease, especially using orMETase instead of injectable rMETase which can cause immunological reactions (31), unlike o-rMETase (28), and will therefore, be suitable clinically in the near future for TNBC.

\section{Conflicts of Interest}

None to be declared. 
A
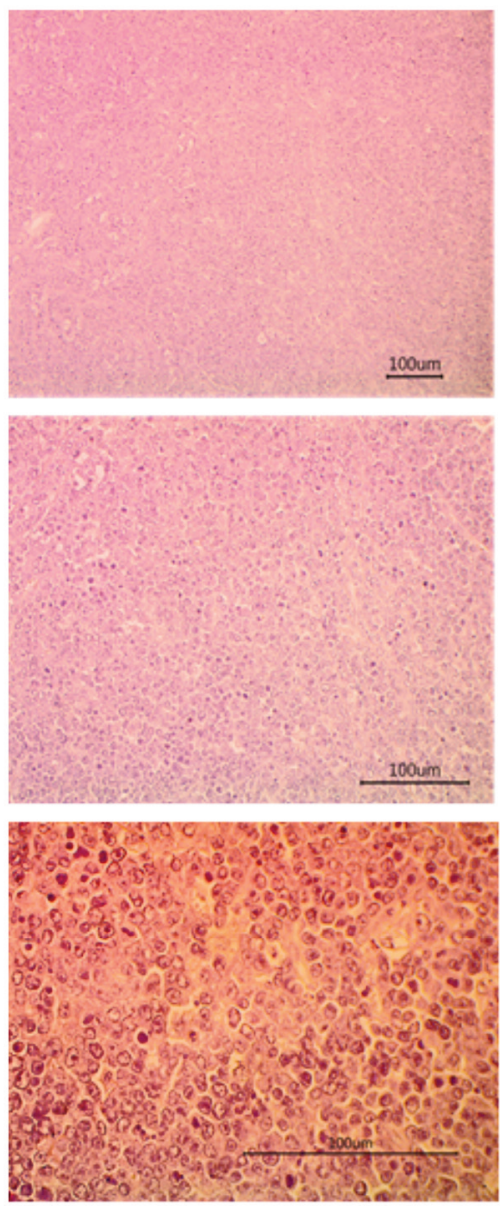

B
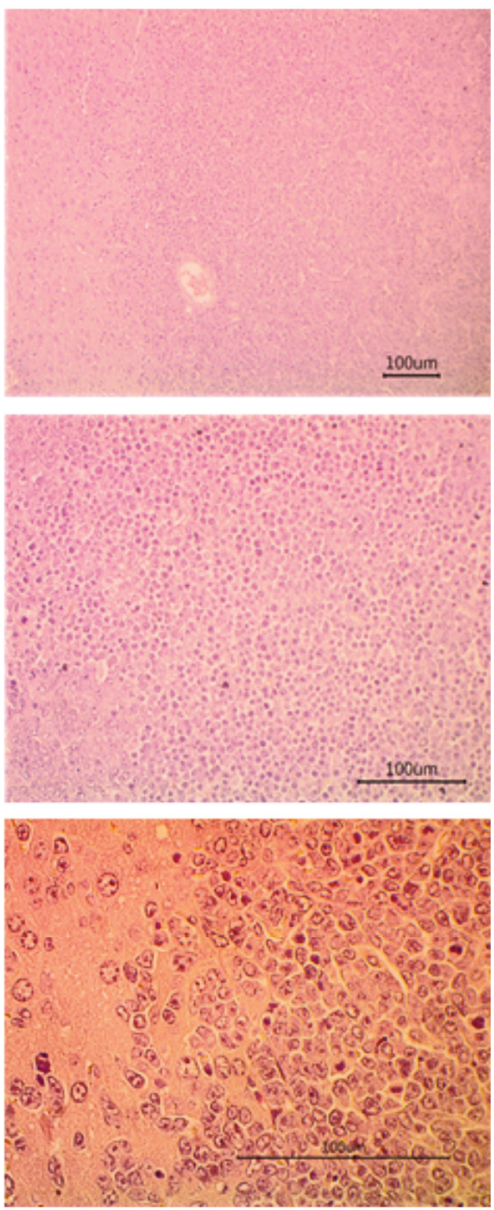

Figure 7. Histology of PDOX TNBC. (A) H\&E staining of mesenteric lymph-node re-metastasis. (B) H\&E staining of SHI tumor. Upper row: $\times 100$, middle row: $\times 200$, lower row: $\times 400$. PDOX: Patient-derived orthotopic xenograft; TNBC: triple-negative breast cancer; H\&E: hematoxylin and eosin.

\section{Authors' Contributions}

HIL and RMH conceived the project. HJC, SJN and MB provided scientific advice for the project. HIL, JY, YS, HN, YT, and NS contributed to mouse-model establishment and obtained experimental data. QH and YT provided methioninase. HIL and $\mathrm{RMH}$ wrote and revised the manuscript.

\section{Acknowledgements}

This paper is dedicated to the memory of AR Moossa MD, Sun Lee, MD, Professor Li Jia Xi and Masaki Kitajima, MD.

\section{References}

1 Dent R, Trudeau M, Pritchard KI, Hanna WM, Kahn HK, Sawka CA, Lickley LA, Rawlinson E, Sun P and Narod SA: Triplenegative breast cancer: Clinical features and patterns of recurrence. Clin Cancer Res 13(15): 4429-4434, 2007. PMID: 17671126. DOI: 10.1158/1078-0432.Ccr-06-3045

2 Hoffman RM: Development of recombinant methioninase to target the general cancer-specific metabolic defect of methionine dependence: A 40-year odyssey. Expert Opin Biol Ther 15(1): 2131, 2015. PMID: 25439528. DOI: 10.1517/14712598.2015.963050

3 Hoffman RM and Erbe RW: High in vivo rates of methionine biosynthesis in transformed human and malignant rat cells auxotrophic for methionine. Proc Natl Acad Sci USA 73(5): 1523-1527, 1976. PMID: 179090. DOI: 10.1073/pnas. 73.5 .1523

4 Stern PH and Hoffman RM: Elevated overall rates of transmethylation in cell lines from diverse human tumors. In Vitro 20(8): 663-670, 1984. PMID: 6500606. DOI: 10.1007/bf02619617

5 Coalson DW, Mecham JO, Stern PH and Hoffman RM: Reduced availability of endogenously synthesized methionine for Sadenosylmethionine formation in methionine-dependent cancer cells. Proc Natl Acad Sci USA 79(14): 4248-4251, 1982. PMID: 6289297. DOI: $10.1073 /$ pnas.79.14.4248 
6 Kaiser P: Methionine dependence of cancer. Biomolecules 10(4), 2020. PMID: 32276408. DOI: 10.3390/biom10040568

7 Hoffman RM, Jacobsen SJ, Erbe RW: Reversion to methionine independence in simian virus 40-transformed human and malignant rat fibroblasts is associated with altered ploidy and altered properties of transformation. Proc Natl Acad Sci USA 76(3): 13131317, 1979. PMID: 220612. DOI: 10.1073/pnas.76.3.1313

8 Hoffman RM and Jacobsen SJ: Reversible growth arrest in simian virus 40-transformed human fibroblasts. Proc Natl Acad Sci USA 77(12): 7306-7310, 1980. PMID: 6261250. DOI: 10.1073/pnas.77.12.7306

9 Stern PH and Hoffman RM: Enhanced in vitro selective toxicity of chemotherapeutic agents for human cancer cells based on a metabolic defect. J Natl Cancer Inst 76(4): 629-639, 1986. PMID: 3457200. DOI: 10.1093/jnci/76.4.629

10 Higuchi T, Kawaguchi K, Miyake K, Han Q, Tan Y, Oshiro H, Sugisawa N, Zhang Z, Razmjooei S, Yamamoto N, Hayashi K, Kimura H, Miwa S, Igarashi K, Chawla SP, Singh AS, Eilber FC, Singh SR, Tsuchiya H and Hoffman RM: Oral recombinant methioninase combined with caffeine and doxorubicin induced regression of a doxorubicin-resistant synovial sarcoma in a PDOX mouse model. Anticancer Res 38(10): 5639-5644, 2018. PMID: 30275182. DOI: 10.21873/anticanres.12899

11 Higuchi T, Oshiro H, Miyake K, Sugisawa N, Han Q, Tan Y, Park J, Zhang Z, Razmjooei S, Yamamoto N, Hayashi K, Kimura H, Miwa S, Igarashi K, Bouvet M, Chawla SP, Singh SR, Tsuchiya $\mathrm{H}$ and Hoffman RM: Oral recombinant methioninase, combined with oral caffeine and injected cisplatinum, overcome cisplatinum-resistance and regresses patient-derived orthotopic xenograft model of osteosarcoma. Anticancer Res 39(9): 46534657, 2019. PMID: 31519563. DOI: 10.21873/anticanres.13646

12 Kawaguchi K, Miyake K, Han Q, Li S, Tan Y, Igarashi K, Kiyuna T, Miyake M, Higuchi T, Oshiro H, Zhang Z, Razmjooei S, Wangsiricharoen S, Bouvet M, Singh SR, Unno M and Hoffman RM: Oral recombinant methioninase (o-rMETase) is superior to injectable rMETase and overcomes acquired gemcitabine resistance in pancreatic cancer. Cancer Lett 432: 251-259, 2018. PMID: 29928962. DOI: 10.1016/j.canlet.2018.06.016

13 Park JH, Zhao M, Han Q, Sun Y, Higuchi T, Sugisawa N, Yamamoto J, Singh SR, Clary B, Bouvet M and Hoffman RM: Efficacy of oral recombinant methioninase combined with oxaliplatinum and 5-fluorouracil on primary colon cancer in a patient-derived orthotopic xenograft mouse model. Biochem Biophys Res Commun 518(2): 306-310, 2019. PMID: 31421825. DOI: $10.1016 /$ j.bbrc.2019.08.051

14 Oshiro H, Tome Y, Kiyuna T, Yoon SN, Lwin TM, Han Q, Tan Y, Miyake K, Higuchi T, Sugisawa N, Katsuya Y, Park JH, Zang Z, Razmjooei S, Bouvet M, Clary B, Singh SR, Kanaya F, Nishida $\mathrm{K}$ and Hoffman RM: Oral recombinant methioninase overcomes colorectal-cancer liver metastasis resistance to the combination of 5-fluorouracil and oxaliplatinum in a patient-derived orthotopic xenograft mouse model. Anticancer Res 39(9): 4667-4671, 2019. PMID: 31519565. DOI: 10.21873/anticanres.13648

15 Kawaguchi K, Igarashi K, Li S, Han Q, Tan Y, Kiyuna T, Miyake K, Murakami T, Chmielowski B, Nelson SD, Russell TA, Dry SM, Li Y, Unno M, Eilber FC and Hoffman RM: Combination treatment with recombinant methioninase enables temozolomide to arrest a BRAF V600E melanoma in a patient-derived orthotopic xenograft (PDOX) mouse model. Oncotarget 8(49): 85516-85525, 2017. PMID: 29156737. DOI: 10.18632/oncotarget.20231
16 Kawaguchi K, Higuchi T, Li S, Han Q, Tan Y, Igarashi K, Zhao M, Miyake K, Kiyuna T, Miyake M, Ohshiro H, Sugisawa N, Zhang Z, Razmjooei S, Wangsiricharoen S, Chmielowski B, Nelson SD, Russell TA, Dry SM, Li Y, Eckardt MA, Singh AS, Singh SR, Eilber FC, Unno M and Hoffman RM: Combination therapy of tumor-targeting Salmonella typhimurium A1-R and oral recombinant methioninase regresses a BRAF-V600E-negative melanoma. Biochem Biophys Res Commun 503(4): 3086-3092, 2018. PMID: 30166061. DOI: 10.1016/j.bbrc.2018.08.097

17 Rashidi B, Sun FX, Jiang P, An Z, Gamagami R, Moossa AR and Hoffman RM: A nude mouse model of massive liver and lymph node metastasis of human colon cancer. Anticancer Res 20(2a): 715-722, 2000. PMID: 10810345.

18 Rashidi B, Gamagami R, Sasson A, Sun FX, Geller J, Moossa AR and Hoffman RM: An orthotopic mouse model of remetastasis of human colon cancer liver metastasis. Clin Cancer Res 6(6): 2556-2561, 2000. PMID: 10873112.

19 Kuo TH, Kubota T, Watanabe M, Furukawa T, Teramoto T, Ishibiki K, Kitajima M, Moossa AR, Penman S and Hoffman RM: Liver colonization competence governs colon cancer metastasis. Proc Natl Acad Sci USA 92(26): 12085-12089, 1995. PMID: 8618849. DOI: 10.1073/pnas.92.26.12085

20 Lim HI, Yamamoto J, Inubushi S, Nishino H, Tashiro Y, Sugisawa N, Han Q, Sun Y, Choi HJ, Nam SJ, Kim MB, Lee JS, Hozumi C, Bouvet M, Singh SR and Hoffman RM: A single low dose of eribulin regressed a highly aggressive triplenegative breast cancer in a patient-derived orthotopic xenograft model. Anticancer Res 40(5): 5, 2020. PMID: 32366392. DOI: 10.21873/anticanres.14218

21 Tan Y, Xu M, Tan X, Tan X, Wang X, Saikawa Y, Nagahama T, Sun X, Lenz M and Hoffman RM: Overexpression and largescale production of recombinant L-methionine-alpha-deaminogamma-mercaptomethane-lyase for novel anticancer therapy. Protein Expr Purif 9(2): 233-245, 1997. PMID: 9056489. DOI: 10.1006/prep.1996.0700

22 Miyake K, Higuchi T, Oshiro H, Zhang Z, Sugisawa N, Park JH, Razmjooei S, Katsuya Y, Barangi M, Li Y, Nelson SD, Murakami T, Homma Y, Hiroshima Y, Matsuyama R, Bouvet M, Chawla SP, Singh SR, Endo I and Hoffman RM: The combination of gemcitabine and docetaxel arrests a doxorubicinresistant dedifferentiated liposarcoma in a patient-derived orthotopic xenograft model. Biomed Pharmacother 117: 109093 , 2019. PMID: 31200257. DOI: 10.1016/j.biopha.2019.109093

23 Ullman-Culleré MH and Foltz CJ: Body condition scoring: A rapid and accurate method for assessing health status in mice. Lab Anim Sci 49(3): 319-323, 1999. PMID: 19619413.

24 Paster EV, Villines KA and Hickman DL: Endpoints for mouse abdominal tumor models: Refinement of current criteria. Comp Med 59(3): 234-241, 2009. PMID: 10403450.

25 Goddard ET, Fischer J and Schedin P: A portal vein injection model to study liver metastasis of breast cancer. J Vis Exp 118, 2016. PMID: 28060292. DOI: $10.3791 / 54903$

26 Jeon H, Kim JH, Lee E, Jang YJ, Son JE, Kwon JY, Lim TG, Kim S, Park JHY, Kim JE and Lee KW: Methionine deprivation suppresses triple-negative breast cancer metastasis in vitro and in vivo. Oncotarget 7(41): 67223-67234, 2016. PMID: 27579534. DOI: $10.18632 /$ oncotarget. 11615

27 Strekalova E, Malin D, Good DM and Cryns VL: Methionine Deprivation Induces a Targetable Vulnerability in TripleNegative Breast Cancer Cells by Enhancing TRAIL Receptor-2 
Expression. Clin Cancer Res 21(12): 2780-2791, 2015. PMID: 25724522. DOI: $10.1158 / 1078-0432$.CCR-14-2792

28 Han Q, Tan Y and Hoffman RM: Oral dosing of recombinant methioninase is associated with a 70\% drop in PSA in a patient with bone-metastatic prostate cancer and $50 \%$ reduction in circulating methionine in a high-stage ovarian cancer patient. Anticancer Res 40(5): 2813-2819, 2020. PMID: 32366428. DOI: 10.21873/anticanres.14254

29 Kawaguchi K, Han Q, Li S, Tan Y, Igarashi K, Murakami T, Unno $M$ and Hoffman RM: Efficacy of recombinant methioninase (rMETase) on recalcitrant cancer patient-derived orthotopic xenograft (PDOX) mouse models: A review. Cells 8(5), 2019. PMID: 31052611. DOI: 10.3390/cells8050410

30 Lim HI, Hamada K, Yamamoto J, Han Q, Tan Y, Choi HJ, Nam SJ, Bouvet $\mathrm{M}$ and Hoffman RM: Oral methioninase inhibits recurrence in a PDOX mouse model of aggressive triplenegative breast cancer. In Vivo 34: 2281-2286, 2020. PMID: 32871751. DOI: 10.21873/invivo.12039
31 Yang Z, Wang J, Lu Q, Xu J, Kobayashi Y, Takakura T, Takimoto A, Yoshioka T, Lian C, Chen C, Zhang D, Zhang Y, Li S, Sun X, Tan Y, Yagi S, Frenkel EP and Hoffman RM: PEGylation confers greatly extended half-life and attenuated immunogenicity to recombinant methioninase in primates. Cancer Res 64(18): 6673-6678, 2204. PMID: 15374983. DOI: 10.1158/0008-5472.CAN-04-1822.
Received July 8, 2020

Revised September 11, 2020

Accepted September 14, 2020 